\title{
Day-ahead optimal dispatching of AC/DC hybrid system
}

\author{
Kai Yuan ${ }^{1}$, Chongbo Sun ${ }^{1}$, Yi Song ${ }^{1}$, and Shigong Jiang ${ }^{1}$ \\ ${ }^{1}$ State Grid Economic and Technological Research Institute Co. Ltd., Beijing, China
}

\begin{abstract}
In this paper, a distributed energy resource (DER) optimal scheduling method for AC/DC hybrid systems with power electronic transformer (PET) is proposed. Firstly, a PET scheduling model considering loss is established. Secondly, an optimal scheduling model of the AC/DC hybrid system with PET is established. Finally, the optimal scheduling model of the AC/DC system with PET is verified by a case and the effect of PET efficiency on the operating state of the system is discussed. The results show that using the power regulation ability of PET, the distributed generation can be fully absorbed and the operating cost of the system can be reduced.
\end{abstract}

\section{Introduction}

In recent years, with the continuous development of distributed generation technology, more and more clean energy has been applied in power system ${ }^{[1]}$. Distributed energy resources (DER) access to the power grid is divided into two types: AC access and DC access. Compared with AC access, DC access can save the commutation link and reduce the system loss. Therefore, the hybrid system with both AC and DC will be one of a dominant system structure in the furture ${ }^{[2]}$. As the distributed power generation device is constrained by various conditions of solar energy and wind energy, its power generation is intermittent, and a large amount of access will cause disturbance to the power grid.

In order to solve this problem, this paper applies power electronic transformer (PET) to AC/DC hybrid system. PET realizes its functions through the combination of power electronic devices and highfrequency transformers. Since the PET has the function of voltage transformation, isolation and energy transmission ${ }^{[3]}$. The structural topology of the AC/DC hybrid system with PET is shown in Figure 1.

In [4], the working principle, topological structure and control strategy of PET are elaborated in detail. In [5], the topological structure and control strategy of applying PET to distribution network are proposed. In [6], a unified reduced-order modeling method and a single-stage control strategy of PET for medium and high voltage power grid is proposed. For the day-ahead optimal dispatch of distributed energy in AC/DC hybrid system, an energy management and coordinated control method is proposed in [7] to realize the coordinated control of multisource $\mathrm{AC} / \mathrm{DC}$ microgrid. In [8], an optimal operation model of AC/DC hybrid system is established and an improved black hole algorithm is proposed to optimize the operation of AC/DC hybrid system. In [9], a probabilistic economic dispatching method for the energy management of AC/DC hybrid system is proposed.

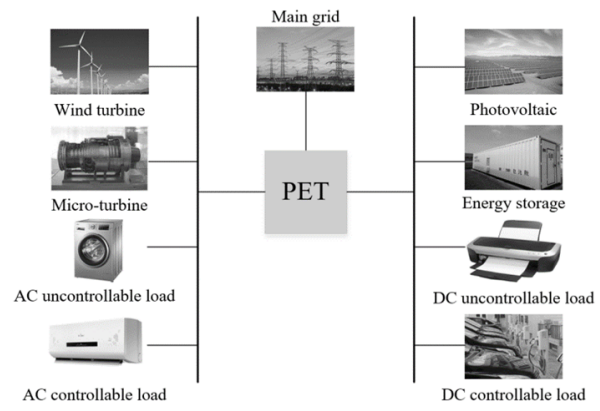

Figure 1. Structure of AC / DC hybrid system with PET

In this paper, an optimal dispatching model is established to verify that the flexible port control ability of PET, which can realize the full absorption of distributed energy and reduce the operating cost of the system.

\section{Model of AC/DC hybrid system with PET}

\subsection{PET optimal scheduling model}

The working principle of PET is shown in Figure $2^{[10]}$. The PET is divided into direct AC/AC type PET and $\mathrm{AC} / \mathrm{DC} / \mathrm{AC}$ type PET, and the latter is commonly used.

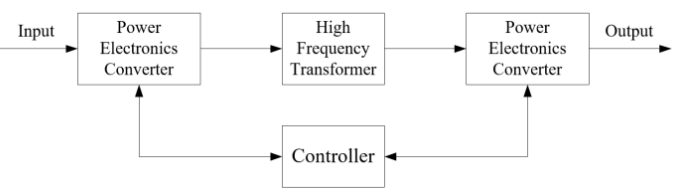

Figure 2. Working principle of PET

\footnotetext{
" Corresponding author: yuankai83@126.com
} 


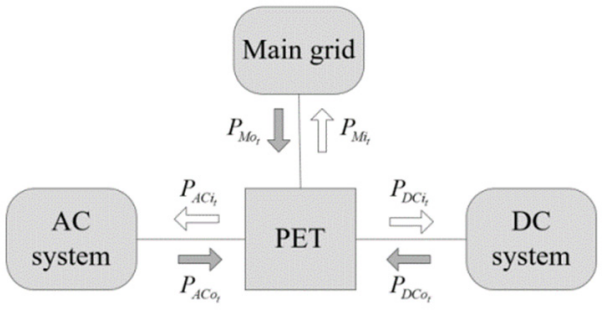

Figure 3. Three-port PET structure

Since the DER power is affected by weather factors, the energy fluctuations in the DC system and the AC system are frequent. PET can coordinate the power flow between the areas and control each port independently ${ }^{[11]}$. Using PET, the main grid can be directly connected to PET, which omits the transformation link between the main gird and the AC system. As the intermediate hub of energy transmission, PET has a certain power loss inside, and defines $\eta$ as the power conversion coefficient of PET. This paper takes three-port PET to establish the scheduling model of PET, whose topology is shown in Figure 3. For the three-port PET, it has:

$$
P_{A C_{t}}+P_{D C i_{t}}+P_{M i_{t}}=\eta\left(P_{A C O_{t}}+P_{D C o_{t}}+P_{M o_{t}}\right)
$$

Where $P_{A C_{i},}, P_{D C_{i}}, P_{M i_{i}}$ are the power flowing from the PET to the $\mathrm{AC}$ area, the $\mathrm{DC}$ area, and the main grid at time $t$, and $P_{A C_{t},}, P_{D C o_{t}}, P_{M o_{t}}$ are the power flowing from the $\mathrm{AC}$ area, the DC area, and the main grid to PET at time $t$, respectively. $P_{A C i_{t}}, P_{D C i_{t}}, P_{M i_{t}}, P_{A C O_{t}}, P_{D C o_{t}}, P_{M o_{t}}$ are positive values. The six positive physical quantities is then reduced to three positive or negative physical quantities:

$$
\begin{gathered}
P_{M}=P_{M o_{t}}-P_{M i_{t}} \\
P_{A C_{t}}=P_{A C O_{t}}-P_{A C i_{t}} \\
P_{D C_{t}}=P_{D C O_{t}}-P_{D C i_{t}}
\end{gathered}
$$

Where $P_{M_{i}}$ represents the interaction power between the main grid and PET at time $t$, and positive value represents the flow of power from the main grid to PET, negative value represents the flow of power from PET to the main grid; $P_{A C_{t}}$ represents the interaction power of the $\mathrm{AC}$ area and PET at time $t$, and positive values represent the flow of power from the AC area to PET, negative values represent the flow of power from PET to the AC area; $P_{D C_{t}}$ represents the interaction power of the DC area and PET at time ${ }^{t}$, and positive values represent the flow of power from the DC area to PET, negative values represent the flow of power from PET to the DC area. The input power of PET can not be determined after simplification, so it is impossible to establish a PET model similar to Eq.(1). This paper solves this problem by introducing the physical quantity of net input power of PET.

$$
\begin{aligned}
P_{P E T_{t}}= & P_{M o_{t}}+P_{A C o_{t}}+P_{D C o_{t}} \\
= & \frac{P_{M o_{t}}+P_{A C o_{t}}+P_{D C o_{t}}+\eta\left(P_{M o_{t}}+P_{A C o_{t}}+P_{D C o_{t}}\right)}{1+\eta} \\
& =\frac{P_{M o_{t}}+P_{A C o_{t}}+P_{D C o_{t}}+P_{M i_{t}}+P_{A C_{t}}+P_{D C_{t}}}{1+\eta} \\
& =\frac{\left|P_{M_{t}}\right|+\left|P_{A C_{t}}\right|+\left|P_{D C_{t}}\right|}{1+\eta}
\end{aligned}
$$

Where $P_{P E T_{t}}$ is the net input power of PET at time $t$, indicating the sum of the total power of PET input from the main grid, the AC area, and the DC area at time ${ }^{t}$. Due to the loss of PET, the following equation holds:

$$
P_{M_{t}}+P_{A C_{t}}+P_{D C_{t}}=(1-\eta) P_{P E T_{t}}
$$

Compare Eq.(5) and Eq.(6), eliminate the net input power $P_{P E T_{t}}$ in the two equations, and the optimal scheduling model of PET is obtained:

$$
P_{M_{t}}+P_{A C_{t}}+P_{D C_{t}}-\frac{1-\eta}{1+\eta}\left(\left|P_{M_{t}}\right|+\left|P_{A C_{t}}\right|+\left|P_{D C_{t}}\right|\right)=0
$$

The following power upper limit constraints for the interaction power of its three ports:

$$
\begin{gathered}
\left|P_{M_{t}}\right| \leq P_{M} \\
\left|P_{A C_{t}}\right| \leq P_{A C} \\
\left|P_{D C_{t}}\right| \leq P_{D C}
\end{gathered}
$$

Where $P_{M}$ is the maximum interaction power between PET and the main grid, $P_{A C}$ is the maximum interaction power between $\mathrm{PET}$ and $\mathrm{AC}$ system, and $P_{D C}$ is the maximum interaction power between PET and DC system.

\subsection{Micro-turbine model}

The power generation constraints of micro-turbine are as follows:

$$
\begin{aligned}
& 0 \leq P_{M T_{t}} \leq P_{M T}^{\max } \\
& P_{M T_{t+1}}-P_{M T_{t}} \leq R U_{M T} \\
& P_{M T_{t}}-P_{M T_{t+1}} \leq R D_{M T}
\end{aligned}
$$

Where $P_{M T_{t}}$ is the output power of the micro-turbine at time $t, P_{M T_{t+1}}$ is the output power of the micro-turbine at time $t+1, P_{M T}^{\max }$ is the maximum output power of the micro-turbine, $R U_{M T}$ is the upper limit of the output power rise rate of the micro-turbine, and $R D_{M T}$ is the upper limit of the output power drop rate of the micro- 
turbine. Eq.(11) limits the power generation of the microturbine, and Eq.(12) and Eq.(13) control the ramp up and fall rates of the micro-turbine.

\subsection{Battery model}

The model of battery systems are expressed as follows:

$$
\begin{aligned}
& P_{B_{t}}=(1-\sigma) P_{B_{t-1}}+P_{B S_{t}} \\
& -P_{D \max } \leq P_{B S_{t}} \leq P_{C \max } \\
& P_{B_{1}}=P_{B_{N}}
\end{aligned}
$$

Where $P_{B_{t}}$ is the stored energy of the battery storage system at time $t, P_{B_{t-1}}$ is the stored energy of the battery storage system at time $t-1, P_{B S_{t}}$ is the charge or discharge amount of the battery storage system at time $t$, and when it is positive, it means that the battery storage system is charged at time ${ }^{t}$; when it is negative, it means that the battery storage system is discharged at time ${ }^{t}$. $\sigma$ is the self-discharge coefficient, $P_{D \max }$ is the upper limit of discharge per unit time of the battery storage system, and $P_{\mathrm{C} \max }$ is the upper limit of charge per unit time of the battery storage system, $P_{B_{1}}$ is the stored energy of the battery storage system at the initial moment, and $P_{B_{N}}$ is the stored energy of the battery storage system at the final moment.

\subsection{Wind turbine model}

This paper assumes that the wind speed satisfies the Weibull distribution, and its probability density function and mathematical expectation are as shown in Eq.(17) and Eq.(18).

$$
\begin{aligned}
& f\left(v_{\text {wind }}\right)=\frac{k}{c}\left(\frac{v_{\text {wind }}}{c}\right)^{k-1} \exp \left[\left(-\frac{v_{\text {wind }}}{c}\right)^{k}\right] \\
& E\left(v_{\text {wind }}\right)=c \times \Gamma\left(1+\frac{1}{k}\right)
\end{aligned}
$$

Where $f(\cdot)$ is the probability density function; $v_{\text {wind }}$ is sample value of the wind speed; $k$ is the shape parameter; $c$ is the scale parameter, and $E(\cdot)$ is the mathematical expectation.

The output power of the wind turbine is calculated according to Eq.(19).

$$
P_{W T}= \begin{cases}0 & v_{\text {wind }}<v_{\text {in }} \\ \frac{v_{\text {wind }}-v_{\text {in }}}{v_{r}-v_{\text {in }}} P_{w t} & v_{\text {in }} \leq v_{\text {wind }}<v_{r} \\ P_{w t} & v_{r} \leq v_{\text {wind }}<v_{\text {out }} \\ 0 & v_{\text {wind }} \geq v_{\text {out }}\end{cases}
$$

Where $P_{W T}$ is the output power of the wind turbine; $v_{\text {in }}, v_{r}$ and $v_{\text {out }}$ are the cut-in wind speed, the rated wind speed and the cut-out wind speed of the wind turbine; $P_{w t}$ is the rated power of the wind turbine.

\subsection{Photovoltaic model}

The output power of photovoltaic satisfies the Beta distribution, and its probability density function and mathematical expectation are as shown in Eq.(20) and Eq.(21).

$$
\begin{gathered}
f\left(\frac{P_{P V}}{P_{P V}^{\max }}\right)=\frac{\Gamma(\alpha+\beta)}{\Gamma(\alpha) \Gamma(\beta)}\left(\frac{P_{P V}}{P_{P V}^{\max }}\right)^{\alpha-1}\left(1-\frac{P_{P V}}{P_{P V}^{\max }}\right)^{\beta-1} \\
E\left(\frac{P_{P V}}{P_{P V}^{\max }}\right)=\frac{\alpha}{\alpha+\beta}=\frac{1}{1+\frac{\beta}{\alpha}}
\end{gathered}
$$

Where $P_{P V}$ and $P_{P V}^{\max }$ are the actual output power and maximum output power of the photovoltaic; $\Gamma(\cdot)$ is the Gamma function; $\alpha$ and $\beta$ are the scale parameters of the Beta distribution. In this paper, $P_{P V}^{\max }$ is regarded as a sampling variable after the standard (the value range is $[0,1])$. When calculating the power output of the photovoltaic, simply multiply the variable by $P_{P V}^{\max }$.

\subsection{Load model}

The load in the system is divided into uncontrollable load and controllable load. For the controllable load, it refers to the load that can be reduced during a certain period of time, such as the air conditioning load, etc. The load model is expressed as follows:

$$
\begin{aligned}
& P_{A C \mathrm{~L}_{t}}=P_{A C \mathrm{Lc}_{t}}+P_{A C \mathrm{Lr}_{t}} \\
& P_{D C \mathrm{~L}_{t}}=P_{D C \mathrm{Lc}_{t}}+P_{D C \mathrm{Lr}_{t}} \\
& P_{A C \mathrm{Lc}_{t}}=P_{A C \mathrm{CC}_{t}}-\Delta_{A C_{t}} \\
& P_{D C \mathrm{Lc}_{t}}=P_{D C \mathrm{CC}_{t}}-\Delta_{D C_{t}} \\
& 0 \leq \Delta_{A C_{t}} \leq \Delta_{A C}^{\max } \\
& 0 \leq \Delta_{D C_{t}} \leq \Delta_{D C}^{\max }
\end{aligned}
$$

Eq.(26) and Eq.(27) ensure that the reduced load is within the allowable range. Where $P_{A C L_{t}}$ is the total load of the AC system at time $t, P_{A C L c_{t}}$ is the controllable load after the load reduction of the AC system at time ${ }^{t}$, $P_{A C \mathrm{Cr}_{i}}$ is the uncontrollable load of the AC system at time $t, P_{A C L C}$ is the controllable load before the load 
reduction of the AC system at time $t, \Delta_{A C_{t}}$ is the reduction load of the AC system at time $t, \Delta_{A C}^{\max }$ is the upper limit of the reduction load of the AC system, $P_{D C L_{\text {, }}}$ is the total load of the DC system at time $t, P_{D C c_{c}}$ is the controllable load after the load reduction of the DC system at time $t, P_{D C \mathrm{r}_{t}}$ is the uncontrollable load of the DC system at time $t, P_{D C C_{1}}$ is the controllable load before the load reduction of the DC system at time ${ }^{t}$, $\Delta_{D C}$ is the reduction load of the DC system at time $t$, and $\Delta_{D C}^{\max }$ is the upper limit of the reduction load of the DC system.

\section{Formulation of the day-ahead optimal scheduling method}

\subsection{Objective function}

An optimal scheduling method of AC/DC hybrid system is proposed with minimum operating cost.

\subsubsection{Equipment maintenance cost}

In this paper, the maintenance cost of wind turbines and photovoltaics is considered, and the maintenance cost of the equipment is related to power. The maintenance cost of them is:

$$
F_{1}=\sum_{t=1}^{N}\left(m_{W T} P_{W T_{t}}+m_{P V} P_{P V_{t}}\right)
$$

Where $N$ is the total number of time of an operation cycle, $m_{W T}$ and $m_{P V}$ are the maintenance cost coefficients of wind turbines and photovoltaics respectively, and $P_{W T_{i}}$ and $P_{P V_{i}}$ are the power generation of wind turbines and photovoltaics at time ${ }^{t}$.

\subsubsection{Micro-turbine power generation cost}

The micro-turbine has a power generation cost, which can be expressed as:

$$
F_{2}=m_{M T} \sum_{t=1}^{N}\left|P_{M T_{t}}\right|
$$

Where $m_{M T}$ is the power generation cost coefficient of the micro-turbine.

\subsubsection{Purchase and sale of electricity cost}

The AC/DC hybrid system is also connected to the main grid. When there is a surplus of power in $\mathrm{AC} / \mathrm{DC}$ system, it can be profited by selling the surplus power to the main grid. When the power in $\mathrm{AC} / \mathrm{DC}$ system is insufficient, the power shortage of the AC/DC system can be balanced by purchasing electricity from the main grid. The total cost of purchasing and selling electricity is the sum of the cost of purchasing electricity and the profit of selling electricity:

$$
\begin{cases}F_{3}=C_{b u y y}+C_{\text {sell }} & \\ C_{b u y}=\sum_{t=1}^{N} m_{M b u y_{t}} P_{M_{t}} & P_{M_{t}} \geq 0 \\ C_{\text {sell }}=\sum_{t=1}^{N} m_{M \text { Mell }} P_{M_{t}} & P_{M_{t}}<0\end{cases}
$$

Where $C_{b u y}$ is the total electricity purchase cost, $C_{\text {sell }}$ represents the total electricity sales cost, $C_{b u y}$ is a positive value, $C_{\text {sell }}$ is a negative value, $m_{M b u y_{t}}$ is the main grid purchase coefficient at time ${ }^{t}$, and $m_{\text {Msell }_{t}}$ is the main grid sales coefficient at time ${ }^{t}$.

\subsubsection{Battery storage loss cost}

During the charging and discharging process of the battery storage system, there is a loss of electrical energy. The cost of battery storage loss is expressed as:

$$
F_{4}=\sum_{i=1}^{N} m_{B} \cdot\left|P_{B_{t}}\right|
$$

Where $m_{B}$ is the loss cost coefficient of the battery storage system.

\subsubsection{Controllable load reduction cost}

The controllable load in the system can be reduced. This cost includes the cost of controllable AC load and DC load at time $t$.

$$
F_{5}=\sum_{t=1}^{N}\left[m_{\Delta_{t}} \cdot\left(\Delta_{A C_{t}}+\Delta_{D C_{t}}\right)\right]
$$

Where $m_{\Delta_{L}}$ is the unit cost of the controllable load at time $t$.

In summary, the objective function of the day-ahead optimal scheduling method is:

$$
\min f=\sum_{i=1}^{5} F_{i}
$$

\subsection{Constraints}

The system should meet the power balance constraints during operation.

$$
P_{A C_{t}}=P_{M T_{s}}+P_{W T_{t}}-P_{A C \mathrm{~L}_{t}}
$$




$$
P_{D C_{t}}=P_{P V_{t}}-P_{B S_{t}}-P_{D \mathrm{CL}_{t}}
$$

Bring Eq.(34) and Eq.(35) into Eq.(7), and the power balance constraints of the system can be obtained:

$$
\begin{aligned}
& P_{M_{t}}+P_{M T_{t}}+P_{W T_{t}}-P_{A C \mathrm{~L}_{t}}+P_{P V_{t}}-P_{B S_{t}}-P_{D C \mathrm{~L}_{t}} \\
& -\frac{1-\eta}{1+\eta}\left(\left|P_{M_{t}}\right|+\left|P_{M T_{t}}+P_{W T_{t}}-P_{A C \mathrm{~L}_{t}}\right|\right. \\
& \left.+\left|P_{P V_{t}}-P_{B S_{t}}-P_{D C \mathrm{~L}_{t}}\right|\right)=0
\end{aligned}
$$

For the constraints of PET, micro-turbines, battery storage system and controllable load, it has been mentioned in Eq.(8)-Eq.(16) and Eq.(22)-Eq.(27).

\section{Case studies}

\subsection{Case study}

A test case of AC/DC hybrid system with PET is shown in Figure 4. The system consists of an AC system and a DC system, which rely on PET for energy transmission. The AC system includes micro-turbine (MT), wind turbine (WT), AC controllable load (AC Lc), and AC uncontrolled load (AC Lr). The amount of power generated by the MT and the amount of reduction of the $\mathrm{AC}$ controllable load at each time are control variables, and the load of the wind turbine and the load of the AC uncontrollable load are given values. The DC system includes photovoltaic (PV), battery storage system (BS), controllable DC load (DC Lc), and DC uncontrollable load (DC Lr).

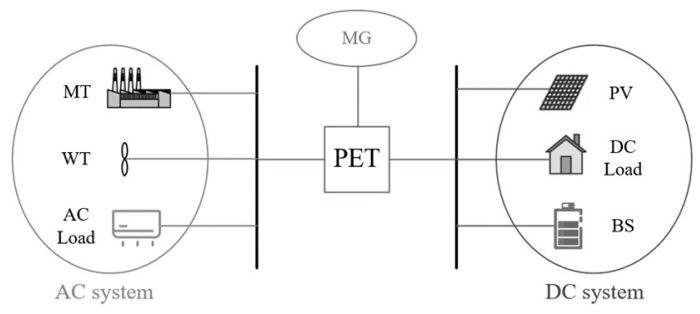

Figure 4. Case of AC/DC hybrid system with PET

The optimal scheduling step length is taken as $1 \mathrm{~h}$, that is, $N=24$. The equipment configuration parameters of the system and the cost coefficients of each part are as follows: The maximum output per unit time of micro-turbine $P_{M T}^{\max }=150 \mathrm{~kW}$, and the ramping power upper limit $R U_{M T}$ $=60 \mathrm{~kW}$, lower power limit $R D_{M T}=60 \mathrm{~kW}$, power generation cost coefficient $m_{M T}=0.45 \mathrm{yuan} /(\mathrm{kW} \cdot \mathrm{h})$. The capacity of battery storage is $200 \mathrm{~kW} \cdot \mathrm{h}$, unit time charging upper limit $P_{C \max }=40 \mathrm{~kW}$, unit time discharge upper limit $P_{D \max }=40 \mathrm{~kW}, \quad$ loss cost coefficient $\quad m_{B}=0.02$ yuan/(kW·h), self-discharge coefficient $\sigma$ is negligible. The maximum output per unit time of the wind turbine is $200 \mathrm{~kW}$, and the equipment maintenance cost coefficient
$m_{W T}=0.025 \mathrm{yuan} /(\mathrm{kW} \cdot \mathrm{h})$. The maximum output per unit time of the photovoltaic is $200 \mathrm{~kW}$, and the equipment maintenance cost coefficient $m_{P V}=0.023$ yuan $/(\mathrm{kW} \cdot \mathrm{h})$. The PET power conversion efficiency $\eta=0.95$. In order to ensure that the $\mathrm{AC} / \mathrm{DC}$ hybrid system can achieve selfsufficiency as much as possible, and also consider the safety and stability of the AC/DC hybrid system, the maximum injection power of the main grid port is limited to $60 \mathrm{~kW}$, and the active injection upper limit values $P_{A C}$ and $P_{D C}$ for the AC system and the DC system port are set to $150 \mathrm{~kW}$. AC controllable load reduction upper limit $\Delta_{A C}^{\max }=50 \mathrm{~kW}$, DC controllable load reduction upper limit $\Delta_{D C}^{\max }=10 \mathrm{~kW}, \mathrm{AC} / \mathrm{DC}$ controllable load reduction cost coefficient $m_{\Delta_{\iota}}=0.55$ yuan $/(\mathrm{kW} \cdot \mathrm{h})$. The 24-hour realtime electricity price data refers to [22].

\subsection{Optimal scheduling results}

Figure 5 shows the output power of AC port and DC port of PET within 24 hours. The positive value indicates that power is flowing from the AC/DC system, and the negative value indicates power is injected into the $\mathrm{AC} / \mathrm{DC}$ system.

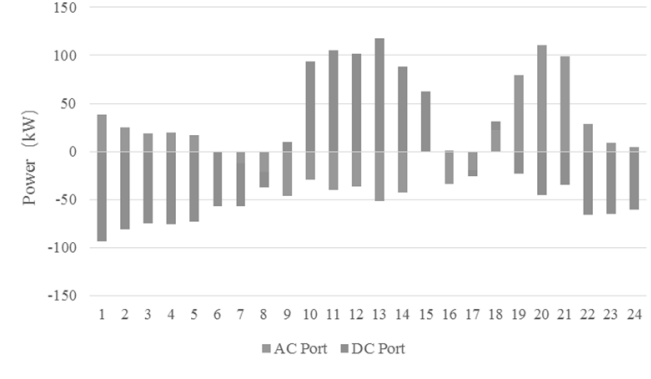

Figure 5. Output power of $\mathrm{AC} / \mathrm{DC}$ port of PET

Take 19-24h as an example, the DC system is in the power shortage state due to the decrease of the PV output. The AC system is in the power surplus state due to the increase of wind speed. At $19-21 \mathrm{~h}$, because MG is at the peak electricity price, the MT of the AC system is fully loaded at this time. The surplus power is transmitted to the DC system and sold to MG for maximum profit. At 22$24 \mathrm{~h}, \mathrm{MG}$ is at the valley electricity price, and the AC/DC systems can purchase electricity from $\mathrm{MG}$ at a lower cost. Therefore, the AC/DC systems purchases electricity from MG at the upper limit of the port at this time period. 


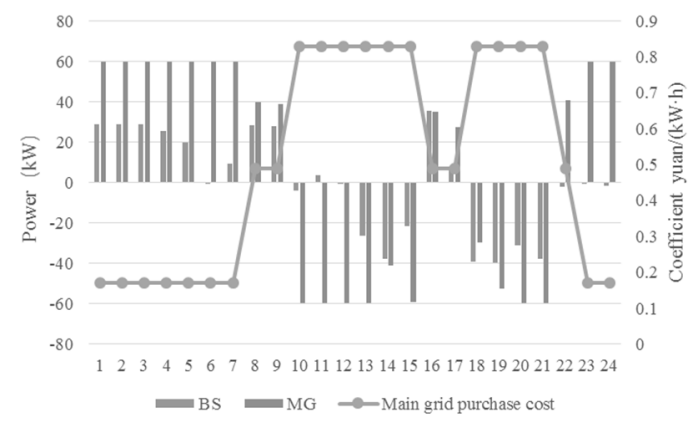

Figure 6. Relationship between battery storage, main grid interaction power and main grid purchase cost

Figure 6 shows the relationship between battery storage, main grid interaction power and main grid purchase cost. When the battery storage is positive, it indicates charging. When it is negative, it indicates discharge. When the main grid interaction power is positive, it indicates power flows from $M G$ to $A C / D C$ systems, and the negative power indicates that the power flows from $\mathrm{AC} / \mathrm{DC}$ systems to MG. It can be clearly seen from Figure 6 that the behavior of the energy exchange between the battery storage and MG has a strong correlation with the main grid purchase cost.

\section{Conclusion}

In this paper, a day-ahead optimal dispatching method for AC/DC hybrid system with PET is proposed to realize the coordinated and optimal operation of "source-loadstorage". By analyzing the optimal dispatching results., it can be seen that the PET can flexibly transmit power in $\mathrm{MG}$, AC system, and DC system through the power regulation capability of its port, thereby improving the absorption capacity of new energy, reducing the operating cost and improving the economy of the system.

\section{Acknowledgements}

This research was funded by the National Key Research and Development Project of China (2018YFB0905000), and the Natural Science Foundation of China (U1966206).

\section{References}

1. Wu Zheng, Kong Li, Yuan Xiaodong, Zhang Shizhong, Yan. Key technologies of AC/DC hybrid renewable energy based on power electronic transformers [J]. New Technology of Electrical Engineering and Energy, 2019, 38 (02): 1-10.

2. Pu Tianjiao, Li Wei, Chen Naishi, Sun Yingyun, $\mathrm{Mu}$ Yunfei, Dong Lei, Kong Li. Key Technology and Research Framework of Optimized Operation Control of AC/DC Hybrid System Based on Power Electronic Transformer[J]. Power System Technology, 2018, 42 (09): 2752-2759.

3. Li Zixin, Gao Fanqiang, Zhao Cong, Wang Zhe, Zhang Hang, Wang Ping, Li Yaohua. Summary of Power Electronic Transformer Technology
Research[J]. Proceedings of the CSEE, 2018, 38(05): 1274-1289.

4. Chen Lei, Ou Jiaxiang, Zhang Qiuyan, et al. Review of research on power electronic transformers [J]. Power Grid and Clean Energy, 2015, 31(12): 36-42.

5. Liao Guohu, Yuan Xufeng, Qiu Guoyue. Simulation Research on Power Electronic Transformer in Distribution Network[J]. Electrical Measurement \& Instrumentation, 2014, 51(17): 35-41.

6. Liu Haijun, Li Gang, Wang Zhikai, et al. Research on modeling method and control strategy of power electronic transformer for medium and high voltage intelligent distribution network[J]. Power System Protection and Control, 2017, 45(02): 85-93.

7. Zhang Xue, Yan, Deng Wei, et al. Energy management and coordinated control method for multi-source/multi-load DC microgrid[J]. Proceedings of the CSEE, 2014, (31): 5553-5562.

8. Di Kaili, Li Xinming, Li Peng, et al. Optimized operation of AC/DC hybrid system based on improved black hole algorithm [J]. Electric Power Construction, 2016, (10): 1-8.

9. Battistelli C, Agalgaonkar Y P, Pal B C. Probabilistic dispatch of remote hybrid microgrids including battery storage and load management $[\mathrm{J}]$. IEEE Transactions on Smart Grid, 2016, PP(99): 1-1.

10. Zhao Zhengming, Feng Gaohui, Yuan Liqiang, Zhang Chunpeng. Development of power router and its key technologies[J]. Proceedings of the CSEE, 2017, 37(13): 3823-3834.

11. Sabahi M, Goharrizi A Y, Hosseini S H, et al. Flexible Power Electronic Transformer [J]. IEEE Transactions on Power Electronics, 2010, 25(8): 2159-2169.

12. $\mathrm{Xu}$ Dongdong. Research on optimal operation method of $\mathrm{AC} / \mathrm{DC}$ hybrid microgrid considering different operation modes [D]. Beijing Jiaotong University, 2017.Title of Site. Available online: URL (accessed on Day Month Year). 